\title{
Microvesicles as Biomarkers in Diabetes, Obesity and Non-Alcoholic Fatty Liver Disease: Current Knowledge and Future Directions
}

\author{
Joshua Welsh ${ }^{\star}$, Judith Holloway and Nicola Englyst
}

Institute of Developmental Sciences (MPT887), University of Southampton Southampton General Hospital, Tremona Road, Southampton SO16 6YD, UK

"Corresponding author: Joshua Welsh, Institute of Developmental Sciences (MPT887), University of Southampton Southampton General Hospital, Tremona Road, Southampton SO16 6YD, UK, Tel: 0238120 6925; E-mail: jw10g13@soton.ac.uk

Received date: September 12, 2014, Accepted date: October 22, 2014, Published date: October 29, 2014

Copyright: (c) 2014 Welsh J, et al. This is an open-access article distributed under the terms of the Creative Commons Attribution License, which permits unrestricted use, distribution, and reproduction in any medium, provided the original author and source are credited.

\begin{abstract}
NAFLD is the most common chronic liver disease, frequently associated with diabetes. Both of these insulin resistant states have increased cardiovascular risk factors associated, and a prevalent cause of mortality in these diseases. Microvesicles are heterogonously sized, phospholipid rich spheres released by cells upon activation and apoptosis. Evidence is continuing to accumulate of microvesicles being not only markers of disease severity but as also having a functional role in the pathophysiology of disease progression.
\end{abstract}

Keywords: Obesity; Diabetes; Fatty liver

\section{What are Microvesicles?}

Microvesicles are shed from the plasma membrane of almost all human cells upon activation and apoptosis (Figure 1).

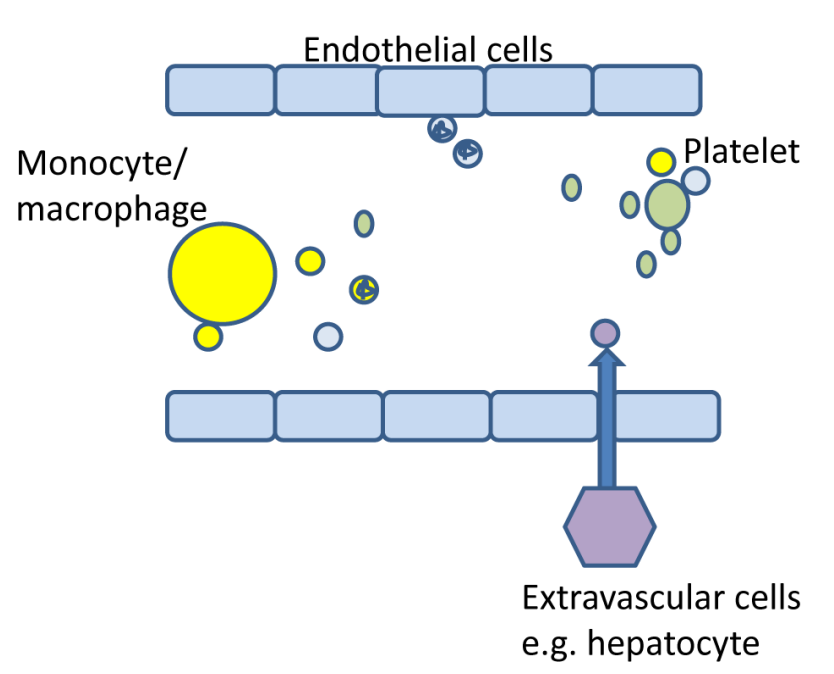

Figure 1: Microvesicles in the blood stream. Microvesicles are released from activated cells within the vasculature but also from distant organs. Each microvesicle carries membrane proteins from the parent cell, allowing identification of the original cell type (represented by different colours). Some carry nuclear material. Microvesicles may interact with each other, with different types of microvesicles or with cells.

A number of stimuli are able to initiate microvesicle release, which is generally associated with an increase in the cell's intracellular $\mathrm{Ca}^{2+}$ concentration. This increase in $\mathrm{Ca}^{2+}$ is thought to modulate enzymes involved in the rearrangement of membrane phospholipids (scramblases, floppases, and flippases) resulting in $\mathrm{Ca}^{2+}$ dependent proteolysis of the membrane cytoskeleton to release microvesicles. Microvesicles therefore express the surface proteins of their parent cell membrane, as well as containing intracellular proteins and/or genetic material. Microvesicles can be found in a wide range of body fluids including, blood, urine, saliva, cerebrospinal fluid and amniotic fluid. Microvesicles are identified predominantly by phosphatidylserine (PS) exposure and/or their diameter, which ranges from 100-1000 nm.

Microvesicles have been implicated in a number of processes including angiogenesis, haemostasis, inflammation, cancer progression and more [1-5]. Due to their size, surface protein expression and intracellular cargo, microvesicles hold the potential to be very interesting biomarkers, with fluctuation in microvesicle numbers and subsets seen in smokers, pregnancy, exercise, obesity, cancer, autoimmune disease, blood disorders, cardiovascular disease, infectious disease, and many more (reviewed by Barteneva et al.) [6].

Microvesicles are released in everyone regardless of disease state, and their numbers reflect the balance between production and clearance. This basal level of microvesicles may be up- or downregulated depending on the situation. Microvesicles have been seen to vary between individuals by as much as 300,000 fold [7]. As well as considering changes in microvesicle counts, disease may change phenotypes and intracellular cargo too. This is likely to be important when considering the utility of MVs as biomarkers. The half-life of microvesicles is considered somewhat variable depending on factors such as size, lipid composition and surface protein expression [8,9]. The half-life of platelet MVs in humans has currently been estimated at less than 6 hours [8]. Little data currently exists for the clearance of microvesicles derived from other sources. However, since one of the main mechanisms in microvesicle clearance is mediated by lactadherin binding, via phosphatidylserine on the microvesicle surface, to macrophages, it should not drastically differ from platelets microvesicles in theory.

Since their identification in 1967, using electron microscopy, our understanding of microvesicle function has made slow progress, particularly due to the lack of accuracy of analytical techniques at the submicron size range. Today, a few options exist for quantification of microvesicle populations including nanoparticle tracking analysis and 
resistive pulse sensing. The most commonly used technique however is flow cytometry. As the flow cytometry field has developed to provide more accurate information for cell analysis, it has inadvertently provided increased accuracy at a submicron level, with modern highend cytometers now capable of analysing a large proportion of the microvesicle population. Flow cytometry also currently holds the edge over other techniques where phenotypic analysis is concerned, as a number of fluorescent labels can simultaneously be analysed per microvesicle.

Platelet MVs are the most abundant MVs, thought to account for around $90 \%$ of circulating MVs, endothelial, granulocyte and monocyte are then the next most common [10,11]. These MVs levels are modulated however depending on the disease and many previous quantification methods have relied on Annexin V binding, which has shown inconsistency in binding between protocols and the type of cell stimulation [12,13]. There is still a need for better development of a general MV marker, as well as applying cell-specific markers (Table 1) to identify where the microvesicles have originated.

\section{Obesity, Diabetes and NAFLD}

Insulin-resistant states such as obesity, type 2 diabetes mellitus (T2DM) and non-alcoholic fatty liver disease (NAFLD) have all been associated with variation in microvesicle subsets, when compared to healthy controls. Those with central obesity are at increased risk of developing insulin resistance, increasing the risk of developing T2DM or NAFLD. Those with T2DM are thought to be more susceptible to developing a more severe form of NAFLD.

Although each of these conditions can exist independently of one another, it appears they are interconnected through insulin resistance. All are associated with multi-organ inflammation, including the vasculature, immune cells and platelets, potentially altering microvesicle production and consequently the impact of microvesicles on cellular behaviour (Figure 2). This is reflected in the microvesicle studies that have been conducted to date, using traditional markers, which show increases in microvesicle numbers derived from the endothelial cells, white blood cells and platelets (Table 1). T2DM is by far the most studied disease, concerning microvesicles, when compared to obesity and NAFLD; studies identifying the involvement of microvesicles with NAFLD to date are particularly lacking.

The main cause of mortality in people with T2DM is cardiovascular disease, and microvesicles released by damaged endothelial cells represent an ideal candidate biomarker for assessing the risk of cardiovascular, as well as microvascular, disease burden in patients with T2DM. Endothelial microvesicles are generally raised; along with platelet microvesicles in patients with T2DM (Table 1).

Platelet metabolism in T2DM is altered and thought to contribute to the atherothrombotic complications associated with the disease [14]. Studies investigating platelet-derived microvesicle markers have fairly consistently shown increases in T2DM patients when compared with healthy individuals (Table 1). This increase in platelet microvesicles could partly account for the hypercoagulable state of the patients, as platelet microvesicles carry procoagulant molecules. Increased microvesicles expressing CD142 (tissue factor) is commonly seen in T2DM patients (Table 1). CD142 is an initiator of coagulation in vivo, and is predominantly expressed on monocytes; with expression also found on platelets and eosinophils [15-17]. In the presence of phosphatidylserine, CD142/factor VII complex activity has been shown to have a 16 million fold increase in activity [18]. It is thought that monocyte/macrophage microvesicles bearing CD142 bind to platelets through P-selectin glycoprotein ligand 1 (PSGL-1), transferring proteins and lipids to the platelet membrane [19]. A functional study has also shown that PSGL-1 is required for CD142+microvesicle accumulation in thrombus formation through CD62P binding in platelets [20]. While convincing, it has also been shown that $\mathrm{CD} 142+$ is not detected within the haemostatic plug at the site of mice skin wounds, and it is therefore unclear whether CD142 and CD142+microvesicles have a role in haemostasis [21]. Further studies into the significance of increased microvesicle-bound CD142 in metabolic diseases, and the functional role it plays, are needed.

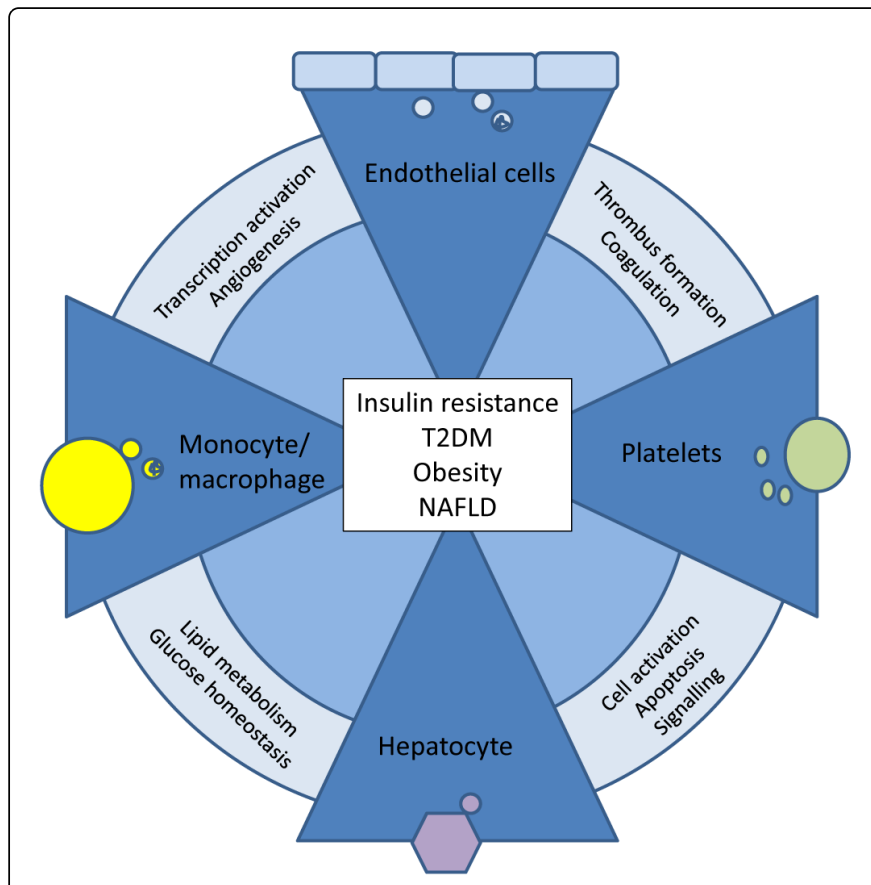

Figure 2: Potential relationships between microvesicles and insulin resistance, obesity, T2DM and NAFLD. Microvesicles produced upon cell activation and apoptosis may alter cellular behaviour in an autocrine or paracrine fashion, to cause changes that favour the development of conditions such as T2DM and NAFLD. Examples of putative microvesicle actions are given. Equally, obesity, insulin resistance, T2DM and NAFLD may influence the production and behaviour of microvesicles.

T2DM is associated with a number of other co-morbidities including kidney disease, retinopathy and neuropathy. Various publications suggest changes in endothelial, platelet and leukocyte microvesicle counts during nepthropathy but there is little published on microvesicle released from kidney-specific cells, or cells related to the other main pathogenic processes in T2DM. This lack of knowledge may offer new potential routes to explore in the future.

Many studies to date have used traditional cell markers, using only a single cell marker to enumerate all microvesicles derived from that cell e.g. CD42 enumerating all platelet-microvesicles. It is however increasingly apparent that microvesicles have varying phenotypes, as do cells, and this phenotype is altered depending upon the stimulant of the parent cell to produce the microvesicle [22-25]. Fitting the full repertoire of parent cell membrane surface markers is also unlikely due to the limited surface area of microvesicles and the area of plasma 
Citation: Welsh J, Holloway J, Englyst N (2014) Microvesicles as Biomarkers in Diabetes, Obesity and Non-Alcoholic Fatty Liver Disease: Current Knowledge and Future Directions. Intern Med S6: S6-009. doi:10.4172/2165-8048.S6-009

Page 3 of 7

membrane they are shed from. Phenotyping of microvesicles is therefore becoming more common but has been overlooked in many of the conducted metabolic studies, which may be failing to observe the functional and translational significance of the increased numbers of microvesicles found in these diseases.

\section{Potential microvesicle biomarkers for the future}

There are a number of molecules carried on microvesicles that may prove to be good biologically relevant biomarkers for diabetes and NAFLD. These include CD36 and Vanin-1, and are discussed in more detail below.

\begin{tabular}{|c|c|c|c|}
\hline \multirow{2}{*}{ Disease } & \multirow{2}{*}{ Procoagulant Marker } & Microvesicle markers & \multirow{2}{*}{ Author } \\
\hline & & $(\uparrow$ increase, $\downarrow$ decrease, $\equiv$ maintained $)$ & \\
\hline \multirow[t]{16}{*}{ T2DM } & Annexin V & $\uparrow \mathrm{CD} 31+/ \mathrm{CD} 42-, \mathrm{CD} 31+/ \mathrm{CD} 42+, \mathrm{CD} 51, \mathrm{CD} 45, \equiv \mathrm{CD} 62 \mathrm{e}$ & Feng et al. $[60,61]$ \\
\hline & Annexin V & $\uparrow$ total microvesicles CD142, CD66e, CD61 & Diamant et al. [62] \\
\hline & N/A & $\uparrow \mathrm{CD} 62 \mathrm{P}, \mathrm{CD} 63$ & Shouzu et al. [62] \\
\hline & N/A & $\uparrow \mathrm{CD} 62 \mathrm{P}, \mathrm{CD} 63, \mathrm{CD} 14$ & Ogata et al. $[63,64]$ \\
\hline & N/A & $\uparrow \mathrm{CD} 31, \mathrm{CD} 105, \mathrm{CD} 106$ & Tramontano et al. [65] \\
\hline & N/A & $\uparrow \mathrm{CD} 42$ & Nomura et al. [66] \\
\hline & Annexin V & $\uparrow$ CD62P, GPIX, CD63, PAC-1, CD14, & Nomura et al. $[51,67]$ \\
\hline & N/A & $\uparrow \mathrm{CD} 144$, 三CD41 & Bernard et al. [68] \\
\hline & N/A & $\uparrow \mathrm{CD} 42 \mathrm{a} / \mathrm{CD} 42 \mathrm{~b}$ & Nomura et al. [54] \\
\hline & N/A & $\uparrow \mathrm{CD} 42 \mathrm{~b}$ & Koga et al. [69] \\
\hline & Annexin $\mathrm{V}$ & $\uparrow \mathrm{CD} 45, \mathrm{CD} 66 \mathrm{~b}, \mathrm{CD} 14$ & Sabatier et al. [70] \\
\hline & N/A & $\uparrow \mathrm{CD} 62 \mathrm{P}, \mathrm{CD} 40$ & Tan et al. [71] \\
\hline & Annexin V & $\uparrow \mathrm{CD} 31+/ \mathrm{CD} 42 \mathrm{~b}-, \mathrm{CD} 31+/ \mathrm{AnV}+$ & Jung et al. [72] \\
\hline & Annexin V & $\uparrow \mathrm{CD} 42 \mathrm{a} / 42 \mathrm{~b}$ & Nomura [73] \\
\hline & Annexin V & $\uparrow \mathrm{CD} 144, \mathrm{CD} 41, \mathrm{CD} 62 \mathrm{P}$ & Tsimerman et al. [74] \\
\hline & Annexin V & $\uparrow \mathrm{CD} 142$ & Sommeijer et al. [75] \\
\hline NAFLD & Annexin V & $\uparrow \mathrm{CD} 14, \downarrow \mathrm{CD} 41, \mathrm{CD} 15$ & Kornek et al. [76] \\
\hline \multirow[t]{5}{*}{ Obesity } & Annexin V & $\uparrow$ Total microvesicles & Goichot et al. [77] \\
\hline & Annexin V & $\uparrow \mathrm{CD} 31, \mathrm{CD} 42 \mathrm{~b}$ & Esposito et al. [78] \\
\hline & N/A & $\uparrow \mathrm{CD} 144$ & Gunduz et al. [79] \\
\hline & Annexin V & $\uparrow$ Total microvesicles, $\uparrow$ CD41+, CD31+/CD41- & Stephanian et al. [80] \\
\hline & $\mathrm{N} / \mathrm{A}$ & $\uparrow \mathrm{CD} 41+$ & Murakami et al. [81] \\
\hline \multicolumn{4}{|c|}{ Platelets Markers - CD41, CD42, CD31, CD61, CD62P } \\
\hline \multicolumn{4}{|c|}{ Endothelial Markers - CD144, CD105, CD106, CD31, CD61, CD62P } \\
\hline \multicolumn{4}{|c|}{ Leukocyte Markers - CD14, CD15 } \\
\hline \multicolumn{4}{|c|}{ Macrophage/Monocyte - CD14, CD15, CD105 } \\
\hline \multicolumn{4}{|c|}{ General Markers - Annexin V, CD63 } \\
\hline
\end{tabular}

Table 1: Microvesicle markers relating to cell identification and procoagulant status when compared with healthy individuals.
CD36: The trans-membrane protein fatty acid translocase (FAT/ CD36) is multifunctional, having effects in lipid metabolism, angiogenesis, atherosclerosis and inflammation. Ligands for CD36 include lipoproteins (HDL, LDL, VLDL, oxidised LDL), long chain fatty acids, and thrombospondin-1 [26-29]. It has a broad expression that includes the endothelium, skeletal muscle, dendritic cells, platelets, monocytes/macrophages, and megakaryocytes [30], which are all are capable of microvesicle shedding. In 2006, a cell-free form of CD36 was identified and named soluble CD36 (sCD36). This has since been associated with atherosclerosis, insulin resistance severity, risk of T2DM, and liver fat [31-33]. 
In 2011, it was suggested that sCD36 is in fact associated with microvesicles in the human plasma of healthy individuals, and is not a cleaved, soluble protein. The study showed sCD36 was particularly associated with platelet-derived microvesicles, as well as leukocytederived and endothelial-derived [34]. These results coincide with the finding that PMICROVESICLEs are elevated in patients with type 2 diabetes (Table 1), along with platelet CD36 activation through lipoproteins, which are also elevated in insulin-resistant states [35-37].

Vanin-1: The imbalance of triglyceride acquisition and removal leads to the range of disorders in NAFLD. NAFLD is often described in four stages starting with simple steatosis, leading on to nonalcoholic steatohepatitis (NASH), fibrosis and finally cirrhosis. At a cellular level this is characterised by intracytoplasmic triglyceridecontaining lipid droplet accumulation in hepatocytes, which can be self-limiting, or progress to hepatocyte ballooning and cell death, with inflammation and/or collagen deposition (fibrosis). From this point hepatocytes are replaced with scar tissue, which consists mainly of collagen I [38].

High-fat diets are capable of causing NAFLD. Recently a novel player in the pathogenesis of NAFLD has been suggested in the form of vanin-1. Vanin (also known as panetetheinase) is an enzyme found in the metabolic pathway of co-enzyme $\mathrm{A}$, and has three isoforms in humans (Vanin-1,2,3) [39]. In humans, Vanin-1 is expressed in the spleen, small intestine, peripheral blood leukocytes and liver. Other forms of vanin also exist (Vanin-2 and 3) and are expressed throughout the body. Vanin hydrolyses pantetheine to form pentethenic acid (Vitamin B5) and cysteamine which is a potent antioxidant. Currently the product of vanin-1 activity, cysteamine, is undergoing clinical trials in NAFLD patients. Cysteamine has been hypothesised to increase reactive oxygen species (ROS) scavenging and glutathione production, thereby improving alanine transaminase (ALT) and aspartate aminotransferase (AST), as well as, inhibiting transgluatminase activity possibly decreasing fibrin crosslinking and therefore inhibiting fibrosis [40].

In vitro studies have shown vanin-1 to be upregulated preceding lipid accumulation and show vanin-1 to be differentially mediated by a number of free fatty acids [41]. Further studies have identified Vanin-1 as an important activator in hepatic gluconeogenesis, having been shown to be involved in the development of hyperglycaemia through increased gluconeogenic gene expression and hepatic glucose out, in loss- and gain-of-function studies [42]. These studies showed transcription to be activated by peroxisome proliferator-activated receptor $\gamma$ coactivator $1 \alpha$ along with hepatocyte nuclear factor $4 \alpha$.

Separate studies have also shown the regulation of vanin-1 by PPAR $a$ to be extremely sensitive, and also identify vanin-1 to be a regulator of hepatic triglyceride levels. Moreover serum vanin-1 has been put forward as a reliable indicator of PPAR $\alpha$ activity in the liver $[43,44]$.

Interestingly, microvesicle bound vanin-1 has been implicated in the promotion of angiogenesis during steatohepatitis, specifically on hepatocyte- microvesicles [45]. Hepatocyte-microvesicles isolated from medium conditioned by murine hepatocytes, as well as a human hepatocyte cell line that had been exposed to saturated free fatty acids and were applied to endothelial cell lines. It was found that the hepatocyte-microvesicles induced migration and tube formation, required for angiogenesis, whilst mediums without hepatocytemicrovesicles, either due to inhibition of formation (through caspase 3 inhibitor) or by ultracentrifugation, lacked proangiogenic activity.
These processes were induced through hepatocyte-microvesicle internalisation, this however required hepatocyte-microvesicle vanin-1 expression, which was abundantly expressed on the hepatocytemicrovesicle. Measurement of hepatocyte-microvesicle bound vanin 1 could therefore act as a biomarker in the progression of NAFLD.

In studies using non-cell specific techniques such as microarrays and western blots, Vanin-1 has been suggested as a biomarker of kidney damage in the rat model of type 1 diabetic nephropathy using urine; medium microvesicles are commonly found in [46]. Another study analysing blood samples showed Vanin-1 as a possible discriminator, along with MMP9, in the diagnosis of pancreatic cancer-associated diabetes from type 2 diabetes [47]. Studies specifically targeting cells and/or microvesicles in these mediums are therefore required and will shed light on its source.

\section{Effect of metabolic disease medications on microvesicles}

Insulin resistant patients can be prescribed numerous medications for insulin resistance itself and also for cardiovascular protection. Studies investigating the effects of these medications in insulin resistant patients further demonstrate the use of microvesicles as diagnostic markers of disease severity.

Dihydropyridine $\mathrm{Ca}^{2+}$ antagonists, nifedipine and benedipine have been investigated in normotensive and hypertensive patients with and without T2DM [48,49]. Platelet- and monocyte-microvesicles were highest in the hypertensive T2DM, followed by T2DM normotensive, hypertensive and normotensive, respectively. Following 3 and 6 months of treatment, platelet-, monocyte- and endothelialmicrovesicles were all significantly reduced in the T2DM patients, with no changes seen in non-diabetic patients. In the benedipine study, looking at monocyte- and endothelial-microvesicles, T2DM patients initially had higher numbers of microvesicles than those without T2DM. All microvesicles levels decreased following the dyhydropyridine treatment in T2DM patients. Of the healthy controls treated, only those with anti-oxLDL antibody $<8.4 \mathrm{AcU} / \mathrm{mL}$ showed significant decrease in microvesicles, those $>8.4 \mathrm{AcU} / \mathrm{mL}$ did not.

Thiazolidinediones: a randomised, double-blind, comparatorcontrolled study comparing the effects of thiazolidinediones, ploglitazone and metformin, on EMICROVESICLEs in patients with newly diagnosed T2DM, showed ploglitazone significantly decreased EMICROVESICLEs, with metformin showing no significant difference in microvesicle levels. It is worth noting that endothelial microvesicles were identified as CD31+/CD42-, it is likely a portion of endothelial microvesicles were CD31+/CD42-/CD41+ and therefore platelet microvesicles.

Statins: Patients with insulin-resistant diseases are commonly prescribed 3-hydroxy-3-methylglutaryl coenzyme A (HMG-CoA) reductase inhibitors (also termed statins), which reduce the likelihood of cardiovascular complications. A study investigating the effects of pravastatin on T2DM patients using Annexin V, CD4, CD8, CD14, CD20, CD62e, glycol-A, CD66b markers, showed no significant difference in the number of microvesicles. These microvesicles markers include T cells, B cells, monocyte/macrophages, granulocytes and endothelial cells. Platelet microvesicles did not show any significant change in CD142, they did however show a decrease in expression levels of CD61. This study shows that the likely inhibition of GPIIIa on platelets had a knock on effect to the type of platelet microvesicles that were produced, adding weight to the microvesicles composition being influenced by the type of activation their parent cell 
receives. An in vitro study looking at the effect of simvastatin on endothelial microvesicles has shown increased endothelial microvesicle numbers which is thought to be mediated by inhibiting prenylation via a caspase 8-dependent mechanism [50]. In vivo studies looking at microvesicles have shown simvastatin used in combination with losartan significantly reduced monocyte-, platelet- and endothelial-microvesicles numbers in combination, when compared to losartan alone [51,52].

Sulphonylureas: Glibenclamide, is a general ATP-binding cassette $(A B C)$ transporter inhibitor used to stimulate insulin secretion of pancreatic $\beta$-cells. A study into its effects on monocytes has shown that glibenclamide caused a reduction in the procoagulant activity of cells, decreasing the expression of CD142 and decreasing the number of microvesicles [53].

Alpha-glucoside inhibitors: These prevent the digestion of carbohydrates. Both miglitol and acarbose, have had their effects studied on T2DM patient platelet microvesicles, with both showing significant decreases after 3 months of therapy [54,55].

Fish oils: Eicosapentaenoic acid (EPA) is an omega-3 fatty currently being investigated as a treatment in NAFLD and T2DM. Studies investigating EPAs effects on hyperlipidemic volunteers with diabetes revealed significant decreases in endothelial microvesicles following 6 months of treatment. Non-diabetic patients however, showed no change in endothelial microvesicles concentrations [56-59].

\section{Conclusion}

Whilst it is clear that microvesicles are implicated in numerous aspects of metabolic diseases and hold the potential for use as biomarkers, it is also clear that many routinely prescribed medications, particularly for T2DM, decrease microvesicles in the patients but not healthy controls. More research is required using phenotypic analysis of microvesicle subsets, along with functional studies to determine whether increases in microvesicles and/or their markers are having positive or negative effects in these diseases. Ultimately this will also provide information on whether MVs can be used as specific standalone diagnostic markers for the pathogenesis of certain diseases.

\section{References}

1. Martinez MC, Andriantsitohaina R (2011) Microparticles in angiogenesis: therapeutic potential. Circ Res 109: 110-119.

2. Owens AP 3rd1, Mackman N (2011) Microparticles in hemostasis and thrombosis. Circ Res 108: 1284-1297.

3. Andriantsitohaina R, Gaceb A, Vergori L, Martínez MC (2012) Microparticles as regulators of cardiovascular inflammation. Trends Cardiovasc Med 22: 88-92.

4. Distler JH, Huber LC, Gay S, Distler O, Pisetsky DS (2006) Microparticles as mediators of cellular cross-talk in inflammatory disease. Autoimmunity 39: 683-690.

5. Muralidharan-Chari V, Clancy JW, Sedgwick A, D'Souza-Schorey C (2010) Microvesicles: mediators of extracellular communication during cancer progression. J Cell Sci 123: 1603-1611.

6. Barteneva NS, Fasler-Kan E, Bernimoulin M, Stern JN, Ponomarev ED, et al. (2013) Circulating microparticles: square the circle. BMC Cell Biol 14: 23.

7. Pol E, Coumans F, Grootemaat A, Gardiner C, Sargent I, Harrison P, et al. Particle size distribution of exosomes and microvesicles determined by transmission electron microscopy, flow cytometry, nanoparticle tracking analysis, and resistive pulse sensing. Journal of thrombosis and haemostasis?: JTH. 2014;
8. Rank A, Nieuwland R, Crispin A, Grützner S, Iberer M, et al. (2011) Clearance of platelet microparticles in vivo. Platelets 22: 111-116.

9. Litvack ML, Post M, Palaniyar N (2011) IgM promotes the clearance of small particles and apoptotic microparticles by macrophages. PLoS One 6: e17223.

10. Tesselaar ME, Romijn FP, Van Der Linden IK, Prins FA, Bertina RM, et al. (2007) Microparticle-associated tissue factor activity: a link between cancer and thrombosis? J Thromb Haemost 5: 520-527.

11. Horstman LL, Ahn YS (1999) Platelet microparticles: a wide-angle perspective. Crit Rev Oncol Hematol 30: 111-142.

12. Connor D, Exner T, Ma D, Joseph J. The majority of circulating plateletderived microparticles fail to bind annexin V, lack phospholipiddependent procoagulant activity and demonstrate greater expression of glycoprotein Ib. Thrombosis and haemostasis. 2010;103(5):1044-52.

13. Albanyan A-M, Murphy M, Rasmussen J, Heegaard C, Harrison P. Measurement of phosphatidylserine exposure during storage of platelet concentrates using the novel probe lactadherin: a comparison study with annexin V. Transfusion. 2009;

14. Ferroni P, Basili S, Falco A, Davì G (2004) Platelet activation in type 2 diabetes mellitus. J Thromb Haemost 2: 1282-1291.

15. Moosbauer C, Morgenstern E, Cuvelier SL, Manukyan D, Bidzhekov K, et al. (2007) Eosinophils are a major intravascular location for tissue factor storage and exposure. Blood 109: 995-1002.

16. Panes O, Matus V, Sáez C, Quiroga T. Human platelets synthesize and express functional tissue factor.

17. Egorina EM, Sovershaev MA, Bjørkøy G, Gruber FX, Olsen JO, et al. (2005) Intracellular and surface distribution of monocyte tissue factor: application to intersubject variability. Arterioscler Thromb Vasc Biol 25: 1493-1498.

18. Morrissey JH (2001) Tissue factor: an enzyme cofactor and a true receptor. Thromb Haemost 86: 66-74.

19. Del Conde I, Shrimpton CN, Thiagarajan P, López JA (2005) Tissuefactor-bearing microvesicles arise from lipid rafts and fuse with activated platelets to initiate coagulation. Blood 106: 1604-1611.

20. Falati S, Liu Q, Gross P, Merrill-Skoloff G, Chou J, et al. (2003) Accumulation of tissue factor into developing thrombi in vivo is dependent upon microparticle P-selectin glycoprotein ligand 1 and platelet P-selectin. J Exp Med 197: 1585-1598.

21. Hoffman M, Whinna HC, Monroe DM (2006) Circulating tissue factor accumulates in thrombi, but not in hemostatic plugs. J Thromb Haemost 4: 2092-2093.

22. Jimenez JJ, Jy W, Mauro LM, Soderland C, Horstman LL, et al. (2003) Endothelial cells release phenotypically and quantitatively distinct microparticles in activation and apoptosis. Thromb Res 109: 175-180.

23. Fox JE, Austin CD, Boyles JK, Steffen PK (1990) Role of the membrane skeleton in preventing the shedding of procoagulant-rich microvesicles from the platelet plasma membrane. J Cell Biol 111: 483-493.

24. Sims P, Wiedmer T, Esmon C, Weiss H, Shattil S. Assembly of the platelet prothrombinase complex is linked to vesiculation of the platelet plasma membrane. Studies in Scott syndrome: an isolated defect in platelet procoagulant activity. The Journal of biological chemistry. 1989;264(29):17049-57.

25. Boulanger CM, Scoazec A, Ebrahimian T, Henry P, Mathieu E, et al. (2001) Circulating microparticles from patients with myocardial infarction cause endothelial dysfunction. Circulation 104: 2649-2652.

26. Calvo D, Gómez-Coronado D, Suárez Y, Lasunción MA, Vega MA (1998) Human CD36 is a high affinity receptor for the native lipoproteins HDL, LDL, and VLDL. J Lipid Res 39: 777-788.

27. Endemann G, Stanton LW, Madden KS, Bryant CM, White RT, et al. (1993) CD36 is a receptor for oxidized low density lipoprotein. J Biol Chem 268: 11811-11816.

28. Abumrad N, el-Maghrabi M, Amri E, Lopez E, Grimaldi P. Cloning of a rat adipocyte membrane protein implicated in binding or transport of long-chain fatty acids that is induced during preadipocyte differentiation. 
Homology with human CD36. The Journal of biological chemistry. 1993;268(24):17665-8.

29. Wang H, Wang Z-H, Kong J, Yang M-Y, Jiang G-H, et al. Oxidized lowdensity lipoprotein-dependent platelet-derived microvesicles trigger procoagulant effects and amplify oxidative stress. Molecular medicine (Cambridge, Mass). 2012;18:159-66.

30. Febbraio M, Hajjar D, Silverstein R. CD36: a class B scavenger receptor involved in angiogenesis, atherosclerosis, inflammation, and lipid metabolism. The Journal of clinical investigation. 2001;108(6):785-91.

31. Handberg A, Norberg M, Stenlund H, Hallmans G, Attermann J, et al. (2010) Soluble CD36 (sCD36) clusters with markers of insulin resistance, and high sCD36 is associated with increased type 2 diabetes risk. J Clin Endocrinol Metab 95: 1939-1946.

32. Handberg A, Højlund K, Gastaldelli A, Flyvbjerg A, Dekker JM, et al. (2012) Plasma sCD36 is associated with markers of atherosclerosis, insulin resistance and fatty liver in a nondiabetic healthy population. J Intern Med 271: 294-304.

33. Knøsgaard L, Thomsen SB, Støckel M, Vestergaard H, Handberg A (2014) Circulating sCD36 is associated with unhealthy fat distribution and elevated circulating triglycerides in morbidly obese individuals. Nutr Diabetes 4: e114.

34. Alkhatatbeh MJ, Mhaidat NM, Enjeti AK, Lincz LF, Thorne RF (2011) The putative diabetic plasma marker, soluble CD36, is non-cleaved, nonsoluble and entirely associated with microparticles. J Thromb Haemost 9: 844-851.

35. Adiels M, Olofsson SO, Taskinen MR, Borén J (2008) Overproduction of very low-density lipoproteins is the hallmark of the dyslipidemia in the metabolic syndrome. Arterioscler Thromb Vasc Biol 28: 1225-1236.

36. Englyst NA, Taube JM, Aitman TJ, Baglin TP, Byrne CD (2003) A novel role for CD36 in VLDL-enhanced platelet activation. Diabetes 52: 1248-1255.

37. Kostapanos MS, Elisaf MS (2014) High density lipoproteins and type 2 diabetes: Emerging concepts in their relationship. World J Exp Med 4: $1-6$.

38. Cohen JC, Horton JD, Hobbs HH (2011) Human fatty liver disease: old questions and new insights. Science 332: 1519-1523.

39. Nitto T, Onodera K (2013) Linkage between coenzyme a metabolism and inflammation: roles of pantetheinase. J Pharmacol Sci 123: 1-8.

40. Dohil R, Schmeltzer S, Cabrera BL, Wang T, Durelle J, et al. (2011) Enteric-coated cysteamine for the treatment of paediatric non-alcoholic fatty liver disease. Aliment Pharmacol Ther 33: 1036-1044.

41. Motomura W, Yoshizaki T, Takahashi N, Kumei S, Mizukami Y, et al (2012) Analysis of vanin-1 upregulation and lipid accumulation in hepatocytes in response to a high-fat diet and free fatty acids. J Clin Biochem Nutr 51: 163-169.

42. Chen S, Zhang W, Tang C, Tang X, Liu L, et al. (2014) Vanin-1 is a key activator for hepatic gluconeogenesis. Diabetes 63: 2073-2085.

43. van Diepen JA, Jansen PA, Ballak DB, Hijmans A, Hooiveld GJ, et al. (2014) PPAR-alpha dependent regulation of vanin-1 mediates hepatic lipid metabolism. J Hepatol 61: 366-372.

44. Rommelaere S, Millet V, Gensollen T, Bourges C, Eeckhoute J, et al. (2013) PPARalpha regulates the production of serum Vanin-1 by liver. FEBS Lett 587: 3742-3748.

45. Povero D, Eguchi A, Niesman I, Andronikou N, Jeu X, Mulya A, et al. Lipid-induced toxicity stimulates hepatocytes to release angiogenic microparticles that require Vanin-1 for uptake by endothelial cells. Science signaling. 2013;6(296):ra88.

46. Fugmann T, Borgia B, Révész C, Godó M, Forsblom C, et al. (2011) Proteomic identification of vanin-1 as a marker of kidney damage in a rat model of type 1 diabetic nephropathy. Kidney Int 80: 272-281.

47. Huang H, Dong X, Kang M, Xu B, Chen Y, Zhang B, et al. Novel blood biomarkers of pancreatic cancer-associated diabetes mellitus identified by peripheral blood-based gene expression profiles. The American journal of gastroenterology. 2010;105(7):1661-9.
48. Nomura S, Inami N, Kimura Y, Omoto S, Shouzu A, et al. (2007) Effect of nifedipine on adiponectin in hypertensive patients with type 2 diabetes mellitus. J Hum Hypertens 21: 38-44.

49. Nomura S, Shouzu A, Omoto S, Nishikawa M, Iwasaka T. Benidipine improves oxidized LDL-dependent monocyte and endothelial dysfunction in hypertensive patients with type 2 diabetes mellitus. Journal of human hypertension. 2005;19(7):551-7.

50. Diamant M, Tushuizen ME, Abid-Hussein MN, Hau CM, Böing AN, et al. (2008) Simvastatin-induced endothelial cell detachment and microparticle release are prenylation dependent. Thromb Haemost 100: 489-497.

51. Nomura S, Shouzu A, Omoto S, Nishikawa M, Iwasaka T. Effects of losartan and simvastatin on monocyte-derived microparticles in hypertensive patients with and without type 2 diabetes mellitus. Clinical and applied thrombosis/hemostasis?: official journal of the International Academy of Clinical and Applied Thrombosis/Hemostasis. 2004;10(2): $133-41$.

52. Nomura S, Shouzu A, Omoto S, Nishikawa M, Fukuhara S, et al. (2004) Losartan and simvastatin inhibit platelet activation in hypertensive patients. J Thromb Thrombolysis 18: 177-185.

53. Henriksson CE, Hellum M, Haug KB, Aass HC, Joø GB, et al. (2011) Anticoagulant effects of an antidiabetic drug on monocytes in vitro. Thromb Res 128: e100-106.

54. Nomura S, Omoto S, Yokoi T, Fujita S, Ozasa R, Eguchi N, et al. Effects of miglitol in platelet-derived microparticle, adiponectin, and selectin level in patients with type 2 diabetes mellitus. International journal of general medicine. 2011;4:539-45.

55. Shimazu T, Inami N, Satoh D, Kajiura T, Yamada K, Iwasaka T, et al. Effect of acarbose on platelet-derived microparticles, soluble selectins, and adiponectin in diabetic patients. Journal of thrombosis and thrombolysis. 2009;28(4):429-35.

56. Nomura S, Shouzu A, Omoto S, Inami N, Ueba T, et al. (2009) Effects of eicosapentaenoic acid on endothelial cell-derived microparticles, angiopoietins and adiponectin in patients with type 2 diabetes. J Atheroscler Thromb 16: 83-90.

57. Boulanger CM, Amabile N, Guérin AP, Pannier B, Leroyer AS, et al. (2007) In vivo shear stress determines circulating levels of endothelial microparticles in end-stage renal disease. Hypertension 49: 902-908.

58. Daniel L, Fakhouri F, Joly D, Mouthon L, Nusbaum P, et al. (2006) Increase of circulating neutrophil and platelet microparticles during acute vasculitis and hemodialysis. Kidney Int 69: 1416-1423.

59. Rabelink TJ, de Boer HC, van Zonneveld AJ (2010) Endothelial activation and circulating markers of endothelial activation in kidney disease. Nat Rev Nephrol 6: 404-414.

60. Feng B, Chen Y, Luo Y, Chen M, Li X, et al. (2010) Circulating level of microparticles and their correlation with arterial elasticity and endothelium-dependent dilation in patients with type 2 diabetes mellitus. Atherosclerosis 208: 264-269.

61. Diamant M, Nieuwland R, Pablo RF, Sturk A, Smit JW, et al. (2002) Elevated numbers of tissue-factor exposing microparticles correlate with components of the metabolic syndrome in uncomplicated type 2 diabetes mellitus. Circulation 106: 2442-2447.

62. Shouzu A, Nomura S, Omoto S, Hayakawa T, Nishikawa M, et al. (2004) Effect of ticlopidine on monocyte-derived microparticles and activated platelet markers in diabetes mellitus. Clin Appl Thromb Hemost 10: 167-173.

63. Ogata N, Nomura S, Shouzu A, Imaizumi M, Arichi M, Matsumura M. Elevation of monocyte-derived microparticles in patients with diabetic retinopathy. Diabetes Research and Clinical Practice. 2006;73(3):241248.

64. Ogata N, Imaizumi M, Nomura S, Shozu A. Increased levels of plateletderived microparticles in patients with diabetic retinopathy. 2005;

65. Tramontano AF, Lyubarova R, Tsiakos J, Palaia T, Deleon JR, et al. (2010) Circulating endothelial microparticles in diabetes mellitus. Mediators Inflamm 2010: 250476. 
Citation: Welsh J, Holloway J, Englyst N (2014) Microvesicles as Biomarkers in Diabetes, Obesity and Non-Alcoholic Fatty Liver Disease: Current Knowledge and Future Directions. Intern Med S6: S6-009. doi:10.4172/2165-8048.S6-009

Page 7 of 7

66. Nomura S, Suzuki M, Katsura K, Xie GL, Miyazaki Y, et al. (1995) Platelet-derived microparticles may influence the development of atherosclerosis in diabetes mellitus. Atherosclerosis 116: 235-240.

67. Nomura S, Shouzu A, Omoto S. Effects of losartan and simvastatin on monocyte-derived microparticles in hypertensive patients with and without type 2 diabetes mellitus. 2004;

68. Bernard S, Loffroy R, Sérusclat A, Boussel L, Bonnefoy E, Thévenon C, et al. Increased levels of endothelial microparticles CD144 (VE-Cadherin) positives in type 2 diabetic patients with coronary noncalcified plaques evaluated by multidetector computed tomography (MDCT). Atherosclerosis. 2009;203(2):429-35.

69. Koga H, Sugiyama S, Kugiyama K, Fukushima H, Watanabe K, Sakamoto $\mathrm{T}$, et al. Elevated levels of remnant lipoproteins are associated with plasma platelet microparticles in patients with type-2 diabetes mellitus without obstructive coronary artery disease. European heart journal. 2006;27(7):817-23.

70. Sabatier F, Darmon P, Hugel B, Combes V, Sanmarco M, Velut J-G, et al. Type 1 and type 2 diabetic patients display different patterns of cellular microparticles. Diabetes. 2002;51(9):2840-5.

71. Tan K, Tayebjee M, Lim H, Lip G. Clinically apparent atherosclerotic disease in diabetes is associated with an increase in platelet microparticle levels. Diabetic medicine?: a journal of the British Diabetic Association. 2005;22(12):1657-62.

72. Jung K-H, Chu K, Lee S-T, Bahn J-J, Kim J-H, Kim M, et al. Risk of macrovascular complications in type 2 diabetes mellitus: endothelial microparticle profiles. Cerebrovascular diseases (Basel, Switzerland). 2011;31(5):485-93.

73. Nomura S, Inami N, Shouzu A, Omoto S, Kimura Y, Takahashi N, et al. The effects of pitavastatin, eicosapentaenoic acid and combined therapy on platelet-derived microparticles and adiponectin in hyperlipidemic, diabetic patients. Platelets. 2009;20(1):16-22.

74. Tsimerman G, Roguin A, Bachar A, Melamed E, Brenner B, et al. (2011) Involvement of microparticles in diabetic vascular complications. Thromb Haemost 106: 310-321.

75. Sommeijer DW, Joop K, Leyte A, Reitsma PH, ten Cate H (2005) Pravastatin reduces fibrinogen receptor gpIIIa on platelet-derived microparticles in patients with type 2 diabetes. J Thromb Haemost 3: 1168-1171.

76. Kornek M, Schuppan D (2012) Microparticles: Modulators and biomarkers of liver disease. J Hepatol 57: 1144-1146.

77. Goichot B, Grunebaum L, Desprez D, Vinzio S, Meyer L, et al. (2006) Circulating procoagulant microparticles in obesity. Diabetes Metab 32: 82-85.

78. Esposito K, Ciotola M, Schisano B, Gualdiero R, Sardelli L, et al. (2006) Endothelial microparticles correlate with endothelial dysfunction in obese women. J Clin Endocrinol Metab 91: 3676-3679.

79. Gunduz Z, Dursun I, Tulpar S, Bastug F, Baykan A, et al. (2012) Increased endothelial microparticles in obese and overweight children. J Pediatr Endocrinol Metab 25: 1111-1117.

80. Stepanian A, Bourguignat L, Hennou S, Coupaye M, Hajage D, et al. (2013) Microparticle increase in severe obesity: not related to metabolic syndrome and unchanged after massive weight loss. Obesity (Silver Spring) 21: 2236-2243.

81. Murakami T, Horigome H, Tanaka K, Nakata Y, Ohkawara K, et al. (2007) Impact of weight reduction on production of platelet-derived microparticles and fibrinolytic parameters in obesity. Thromb Res 119: 45-53

This article was originally published in a special issue, entitled: "Obesity \&

Diabetes", Edited by Ippei Kanazawa, Shimane University, Japan 